\title{
An improved assembly and annotation of the allohexaploid wheat genome identifies complete families of agronomic genes and provides genomic evidence for chromosomal translocations
}

\author{
Bernardo J. Clavijo, ${ }^{1,9}$ Luca Venturini, ${ }^{1,9}$ Christian Schudoma, ${ }^{1}$ \\ Gonzalo Garcia Accinelli, ${ }^{1}$ Gemy Kaithakottil, ${ }^{1}$ Jonathan Wright, ${ }^{1}$ Philippa Borrill, ${ }^{2}$ \\ George Kettleborough, ${ }^{1}$ Darren Heavens, ${ }^{1}$ Helen Chapman, ${ }^{1}$ James Lipscombe, ${ }^{1}$ \\ Tom Barker, ${ }^{1}$ Fu-Hao Lu, ${ }^{2}$ Neil McKenzie, ${ }^{2}$ Dina Raats, ${ }^{1}$ Ricardo H. Ramirez-Gonzalez, ${ }^{1,2}$ \\ Aurore Coince, ${ }^{1}$ Ned Peel, ${ }^{1}$ Lawrence Percival-Alwyn, ${ }^{1}$ Owen Duncan, ${ }^{3}$ Josua Trösch, ${ }^{3}$ \\ Guotai Yu, ${ }^{2}$ Dan M. Bolser, ${ }^{4}$ Guy Namaati, ${ }^{4}$ Arnaud Kerhornou, ${ }^{4}$ Manuel Spannagl, ${ }^{5}$ \\ Heidrun Gundlach, ${ }^{5}$ Georg Haberer, ${ }^{5}$ Robert P. Davey, ${ }^{1,6}$ Christine Fosker, ${ }^{1}$ \\ Federica Di Palma, ${ }^{1,6}$ Andrew L. Phillips, ${ }^{7}$ A. Harvey Millar, ${ }^{3}$ Paul J. Kersey, ${ }^{4}$ \\ Cristobal Uauy, ${ }^{2}$ Ksenia V. Krasileva, ${ }^{1,6,8}$ David Swarbreck, ${ }^{1,6}$ Michael W. Bevan, ${ }^{2}$ \\ and Matthew D. Clark ${ }^{1,6}$ \\ ${ }^{1}$ Earlham Institute, Norwich, NR4 7UZ, United Kingdom; ${ }^{2}$ John Innes Centre, Norwich, NR4 7UH, United Kingdom; ${ }^{3}$ ARC Centre of \\ Excellence in Plant Energy Biology, The University of Western Australia, Crawley Western Australia 6009, Australia; ${ }^{4}$ EMBL European \\ Bioinformatics Institute, Hinxton, CB10 1SD, United Kingdom; ${ }^{5}$ Plant Genome and Systems Biology, Helmholtz Center Munich, \\ 85764 Neuherberg, Germany; ${ }^{6}$ University of East Anglia, Norwich, NR4 7TJ, United Kingdom; ${ }^{7}$ Rothamsted Research, Harpenden, \\ AL5 2JQ, United Kingdom; ${ }^{8}$ The Sainsbury Laboratory, Norwich, NR4 7UH, United Kingdom
}

\begin{abstract}
Advances in genome sequencing and assembly technologies are generating many high-quality genome sequences, but assemblies of large, repeat-rich polyploid genomes, such as that of bread wheat, remain fragmented and incomplete. We have generated a new wheat whole-genome shotgun sequence assembly using a combination of optimized data types and an assembly algorithm designed to deal with large and complex genomes. The new assembly represents $>78 \%$ of the genome with a scaffold $\mathrm{N} 50$ of $88.8 \mathrm{~kb}$ that has a high fidelity to the input data. Our new annotation combines strand-specific Illumina RNA-seq and Pacific Biosciences (PacBio) full-length cDNAs to identify 104,091 high-confidence protein-coding genes and 10,156 noncoding RNA genes. We confirmed three known and identified one novel genome rearrangements. Our approach enables the rapid and scalable assembly of wheat genomes, the identification of structural variants, and the definition of complete gene models, all powerful resources for trait analysis and breeding of this key global crop.
\end{abstract}

[Supplemental material is available for this article.]

Improvements in sequencing read lengths and throughput have enabled the rapid and cost-effective assembly of many large and complex genomes (Gnerre et al. 2011; Lam et al. 2011). Comparisons between assembled genomes have revealed many classes of sequence variation of major functional significance that were not detected by direct alignment of sequence reads to a common reference (The 1000 Genomes Project Consortium 2010; Gan et al. 2011; Bishara et al. 2015). Therefore, accurate comparative genomics requires that genome sequences are assembled

\footnotetext{
9These authors contributed equally to this work.

Corresponding authors: matt.clark@earlham.ac.uk, David. Swarbreck@earlham.ac.uk, michael.bevan@jic.ac.uk

Article published online before print. Article, supplemental material, and publication date are at http://www.genome.org/cgi/doi/10.1101/gr.217117.116.

Freely available online through the Genome Research Open Access option.
}

prior to alignment, but in many eukaryotic genomes, assembly is complicated by the presence of large tracts of repetitive sequences (Treangen and Salzberg 2012; Chaisson et al. 2015) and the common occurrence of genome duplications, for example, in polyploids (Blanc and Wolfe 2004; Berthelot et al. 2014).

Recent innovations in sequence library preparation, assembly algorithms, and long-range scaffolding have dramatically improved whole-genome shotgun assemblies from short-read sequences. These include PCR-free library preparation to reduce bias (Aird et al. 2011), longer sequence reads, and algorithms that preserve allelic diversity during assembly (Weisenfeld et al. 2014). Short-read assemblies have been linked into larger

(C) 2017 Clavijo et al. This article, published in Genome Research, is available under a Creative Commons License (Attribution 4.0 International), as described at http://creativecommons.org/licenses/by/4.0/. 
chromosome-scale scaffolds by Hi-C in vivo (Lieberman-Aiden et al. 2009) and in vitro (Putnam et al. 2016) chromatin proximity ligation, as well as by linked-read sequencing technologies (Mostovoy et al. 2016; Weisenfeld et al. 2016). Although it is more expensive than short-read sequencing approaches, singlemolecule real-time (SMRT) sequencing improved the contiguity and repeat representation of mammalian (Pendleton et al. 2015; Gordon et al. 2016; Bickhart et al. 2017) and diploid grass genomes (Zimin et al. 2017). SMRT technologies are also being used to generate the complete sequence of transcripts, increasing the accuracy of splicing isoform definition (Abdel-Ghany et al. 2016).

The assembly of the $17 \mathrm{~Gb}$ allohexaploid genome of bread wheat (Triticum aestivum) has posed major difficulties, as it is composed of three large, repetitive, and closely related genomes (Moore et al. 1995). Despite progressive improvements, an accurate and near-complete wheat genome sequence assembly and corresponding high-quality gene annotation has not yet been generated. Initial whole-genome sequencing used orthologous Poaceae protein sequences to generate highly fragmented gene assemblies (Brenchley et al. 2012). A BAC-based assembly of Chromosome 3B provided major insights into wheat chromosome organization (Choulet et al. 2014). Illumina sequencing and assembly of flow-sorted chromosome arm DNA (Chromosome Survey Sequencing [CSS]) identified homoeologous relationships between genes in the three genomes, but the assemblies remained highly fragmented (The International Wheat Genome Sequencing Consortium 2014). Recently, a whole-genome shotgun sequence of hexaploid wheat was assembled and anchored, though not annotated, using an ultradense genetic map (Chapman et al. 2015). The assembly contained $\sim 48.2 \%$ of the genome with contig and scaffold N50 lengths of 8.3 and $25 \mathrm{~kb}$, respectively.

Here we report the most complete and accurate sequence assembly and annotation to date of the allohexaploid wheat reference accession, Chinese Spring (CS42). Our approach is open source, rapid, and scalable and enables a more in-depth analysis of sequence and structural variation in this key global crop.

\section{Results}

\section{DNA library preparation and sequencing}

We aimed to reduce bias and retain maximum sequence complexity by using unamplified libraries for contig generation (Kozarewa et al. 2009) and to improve scaffolding by using precisely sized mate-pair libraries (Heavens et al. 2015). Libraries were sequenced using Illumina paired-end (PE) 250-bp reads to distinguish closely related sequences. In total, 1.1 billion PE reads were generated to provide $33 \times$ sequence coverage of the CS42 genome (Supplemental Information S1; Supplemental Table S4.1). For scaffolding, long mate-pair (LMP) libraries with insert sizes ranging from 2480-11,600 bp provided 53× sequence coverage, and Tight, Amplification-free, Large insert PE Libraries (TALL) with an insert size of 690 bp provided $15 \times$ sequence coverage (Supplemental Information S1; Supplemental Table S4.2).

\section{Genome assembly}

Nearly 3 million contigs (of length $>500 \mathrm{bp}$ ) were generated using the w2rap-contigger (Clavijo et al. 2017) with an N50 of $16.7 \mathrm{~kb}$ (Supplemental Information S1; Supplemental Table S4.3). After scaffolding using SOAPdenovo (Luo et al. 2012), the assembly contained 1.3 million sequences with an N50 of $83.9 \mathrm{kbp}$. The TGACv1 scaffolds were classified to chromosome arms using raw CSS reads (The International Wheat Genome Sequencing Consortium 2014) and subsequently screened with a two-tiered filter based first on their length and their $k$-mer content (see Supplemental Information S1, section S4.5). The approach removed short, redundant sequences from the assembly minimizing the loss of unique sequence content, leading to an increase in scaffold N50 to $88.8 \mathrm{~kb}$. Contig accuracy was assessed by mapping links from the 11-kb LMP library, which was not used in the contig assembly. Breaks in the linkage at different mate-pair mapping coverages only affected a very small portion of the content and did not reduce N50 contiguity significantly (Supplemental Information S1; Supplemental Figs. S4.4, S4.5). Supplemental Tables S4.5 and S4.6 in S1 show that $91.1 \%$ of TGACv1 genes were correctly assigned to Chromosome $3 \mathrm{~B}$, with no discrepancies in gene order identified.

The genome of a synthetic wheat line W7984 was previously assembled with an improved version of meraculous (Chapman et al. 2011) using 150-bp PE libraries with varying insert sizes, for a combined genome coverage of $34.3 \times$, together with 1.5and 4-kb LMP libraries for scaffolding (Chapman et al. 2015). This contig assembly, with an N50 of $8.3 \mathrm{~kb}$, covered $8 \mathrm{~Gb}$ of the genome while the scaffold assembly covered $8.21 \mathrm{~Gb}$ with an N50 of $24.8 \mathrm{~kb}$. In comparison, the TGACv1 assembly represents $\sim 80 \%$ of the 17 -Gb genome, a $60 \%$ improvement in genome coverage. The contiguity of the TGACv1 assembly, as measured by scaffold N50 values, is 3.7-fold greater than that of the W7984 assembly and 30 times that of the CSS assembly (Table 1; The International Wheat Genome Sequencing Consortium 2014).

A KAT $k$-mer spectra copy number plot provides information to analyze how much and what type of $k$-mer content from reads is present in an assembly (Mapleson et al. 2017). It decomposes the $k$ mer spectrum of a read data set by the frequency in which the $k$ mers are encountered in the assembly. The plot generated from TGACv1 (Fig. 1A) showed that $k$-mers found at low frequency (less than 12), representing sequencing errors, were not found in the assembly (shown by the black distribution at $k$-mer multiplicity less than 12). Most sequence content was represented in the assembly once (shown by the main red distribution), with $k$-mers originating from the repetitive and the homoeologous regions of the genome represented at higher frequencies (more than 50). The absence of $k$-mers in the assembly that are not present in the reads indicated that the assembled contigs accurately reflected the input data. A similar analysis of the CSS assembly (Fig. 1B) identified approximately 50 million $k$-mers that were not found as sequenced content in the PCR-free paired-end data, as shown by the red bar at $k$-mer multiplicity equal to zero. This is indicative of chimeric sequences or consensus inconsistencies in the CSS assembly. The black distribution between $k$-mer multiplicity 15 and 45 shows $k$-mers from the PCR-free reads that were not present in

Table 1. Comparison of TGACv1 scaffolds to the IWGSC and Chapman assemblies of hexaploid wheat

\begin{tabular}{lrcrrrrr}
\hline & $\begin{array}{c}\text { Size } \\
(\mathbf{G b})\end{array}$ & $\begin{array}{c}\text { Seq. } \\
\text { count }\end{array}$ & $\begin{array}{r}\text { N20 } \\
(\mathbf{k b})\end{array}$ & $\begin{array}{r}\text { N50 } \\
(\mathbf{k b})\end{array}$ & $\begin{array}{r}\text { N80 } \\
(\mathbf{k b})\end{array}$ & $\begin{array}{r}\text { \% } \\
\text { Ns }\end{array}$ & $\begin{array}{c}\text { \% of } \\
\text { genome }\end{array}$ \\
\hline TGACV1 & 13.43 & 735,943 & 180.1 & 88.8 & 32.8 & 5.7 & 78.8 \\
W7984 & 8.21 & 955,122 & 47.1 & 24.8 & 9.9 & 15.2 & 48.2 \\
CSS & 8.32 & $4,061,833$ & 8.6 & 3.3 & 1.2 & 1.0 & 48.9 \\
\hline
\end{tabular}

Numbers are calculated using sequences $>500 \mathrm{bp}$ and including gaps (Ns) for each assembly. (IWGSC) International Wheat Genome Sequencing Consortium.

\section{Genome Research}

www.genome.org 

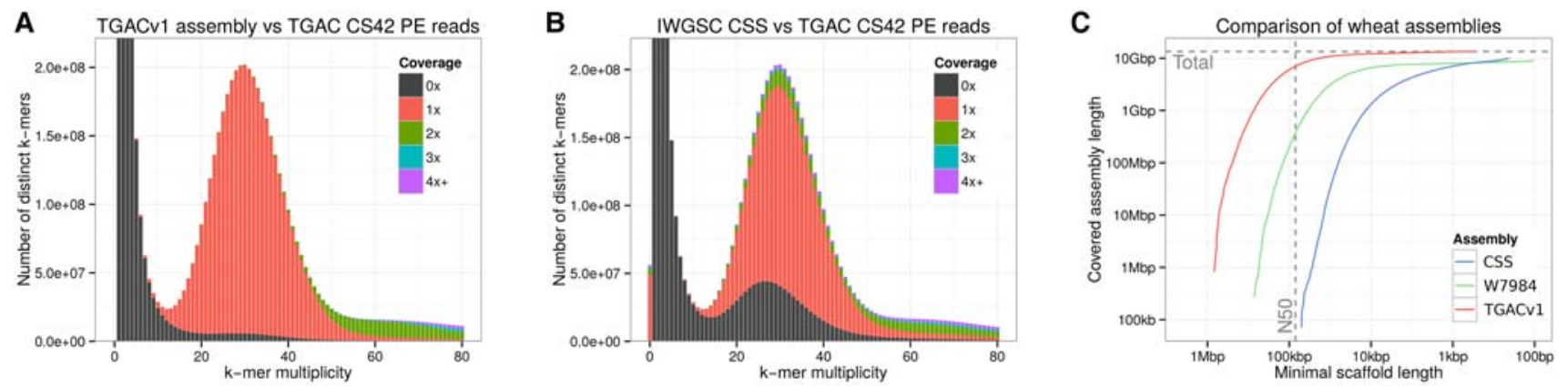

D

Triticum_aestivum_CS42_TGACv1_scaffold_577042_7BL

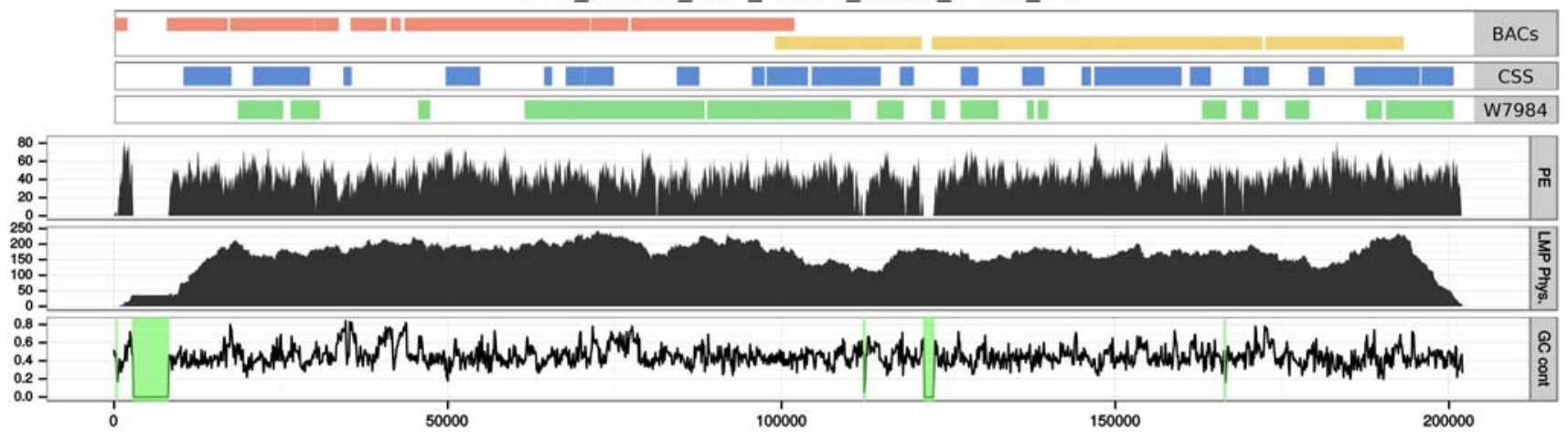

Figure 1. Summary of the TGACv1 wheat genome sequence assembly. $(A, B)$ KAT spectra-cn plots comparing the PE reads to the TGACv1 scaffolds $(A)$ and CSS scaffolds $(B)$. Plots are colored to show how many times fixed length words ( $k$-mers) from the reads appear in the assembly; frequency of occurrence (multiplicity; $x$-axis) and number of distinct $k$-mers ( $y$-axis). Black represents $k$-mers missing from the assembly; red, $k$-mers that appear once in the assembly; green, twice; etc. Plots were generated using $k=31$. The black distribution between $k$-mer multiplicity 15 and 45 in $B$ represents $k$-mers that do not appear in the CSS assembly. (C) Comparison of scaffold lengths and total assembly sizes of the TGACv1, W7984, and CSS assemblies. (D) Scaffold 577042 of the TGACv1 assembly. Tracks from top to bottom: aligned BAC contigs, CSS contigs, W7984 contigs, coverage of PE reads, coverage of LMP fragments, and GC content with scaffolded gaps ( $\mathrm{N}$ stretches) with $0 \% \mathrm{GC}$ highlighted in green. There are two BACs (composed of seven and four contigs each), 22 CSS contigs, and 15 W7984 contigs across the single TGACv1 scaffold.

the CSS assembly, most probably coming from the one-third of the genome not represented by the CSS assembly. The PCR-free library is expected to capture unbiased coverage of the genome, which is reflected in the increased size of the TGACv1 assembly compared with the CSS assembly. Greater amounts of duplication were observed in the single copy regions of the CSS assembly, corresponding to the purple and green areas above the main red distribution.

The content and order of genes in TGACv1 scaffolds assigned to Chromosome 3B (Supplemental Information S1; Supplemental Table S4.4) was compared to that in the Chromosome 3B BACbased assembly (Choulet et al. 2014); $91.2 \%$ of the genes previously identified on the 3B BAC-based assembly aligned to TGACv1 scaffolds (Table 2), with no discrepancies in gene order (Supplemental Information S1; Supplemental Table S4.5). This compared with $73.9 \%$ aligned to W7984 3B scaffolds and $68.0 \%$ aligned to CSS Chromosome 3B scaffolds, demonstrating the improved representation of the TGACv1 assembly.

Alignment of TGACv1 3B scaffolds to the 3B BAC-based pseudomolecule (Fig. 2A,C) showed that they were largely in agreement. Two examples of apparent disagreement are shown in Figure 2, B and D. Scaffold_221671_3B spanned a gap of $700 \mathrm{~kb}$ in the $3 \mathrm{~B}$ BAC assembly, and reoriented and removed a duplication, by identifying both ends of a CACTA element (Fig. 2B). Scaffold_220592_3B spanned $582 \mathrm{~kb}$ and diverged in one location (Fig. 2D) and contained a Sabrina solo-LTR with a characteristic ATCAG target site duplication (TSD). In scaffold_220592_3B, the TSD was present on either side of the Sabrina_3231 element, while in the BAC-based scaffold Sabrina homology ended in Ns. In the BAC-based assembly, only one side of the disjunction showed alignment similarity to CACTA_3026, which was found to be complete in scaffold_220592_3B and spanned the disjunction (Fig. 2D). These two examples illustrate how the TGACv1 assembly generated accurate scaffolds spanning typical complex and long tracts of repetitive DNA characterizing the wheat genome, which were misassembled in the BAC-based approach.

\section{Repetitive DNA composition}

More than $80 \%$ of the $13.4-\mathrm{Gb}$ assembly was composed of approximately 9.7 million annotated transposable element entities, of which $\sim 70 \%$ were retroelements (class I) and 13\% DNA

Table 2. Comparison of TGACv1 Chromosome 3B scaffolds to BACbased scaffolds (Choulet et al. 2014) and 3B scaffolds from the W7984 and CSS assemblies

\begin{tabular}{llrrrr}
\hline & $\begin{array}{c}\text { Scaffold } \\
\text { count }\end{array}$ & $\begin{array}{c}\text { N50 } \\
\text { (kb) }\end{array}$ & $\begin{array}{c}\text { Total seq. } \\
\text { (Mb) }\end{array}$ & $\begin{array}{l}\text { Gene } \\
\text { count }\end{array}$ & \multicolumn{1}{c}{$\begin{array}{c}\text { \% } \\
\text { genes }\end{array}$} \\
\hline 3B ref. & 2808 & 892.4 & 832.8 & 7703 & 100.0 \\
TGACv1 & 29,090 & 116.5 & 790.0 & 6983 & 91.2 \\
W7984 & 26,206 & 30.6 & 479.4 & 5671 & 73.9 \\
CSS & 272,072 & 3.4 & 557.2 & 5233 & 68.0 \\
\hline
\end{tabular}

Numbers are calculated using sequences $>500$ bp and including gaps (Ns) for each assembly. 
A

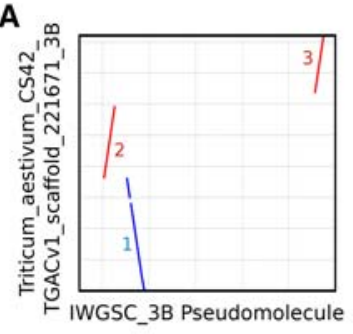

C

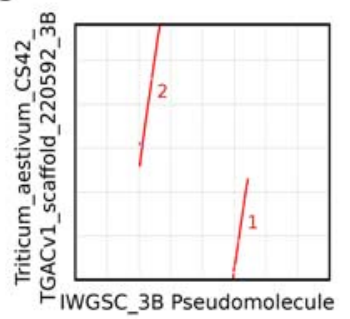

B

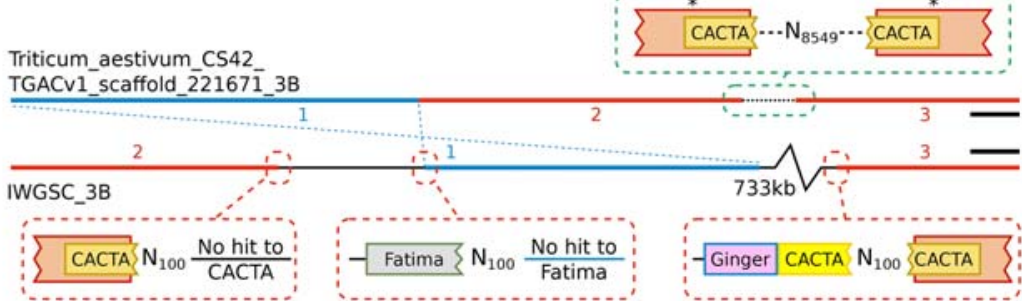

D

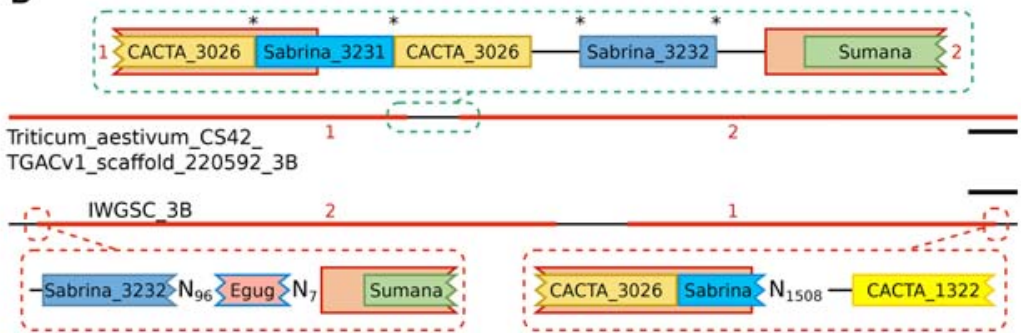

Figure 2. Comparative alignment of TGACv1 scaffolds with the $3 B$ BAC-based pseudomolecule. $(A, C)$ Dot plots between TGACv1 scaffolds and $3 B$ show disruptions in sequence alignment, including rearrangements (red) and inversions (blue). $(B, D)$ Graphical representation of sequence annotations in disrupted regions. Junctions in the TGACv1 scaffolds are consistent with a complete retroelement spanning the junction that includes identical TSD on either side of the retroelement (asterisks). Corresponding regions in the 3B BAC-based pseudomolecule are characterized by Ns that produce inconsistent alignment of retroelements across putative junctions. Retroelements of the same family (CACTA, Sabrina) but matching distinct members in the TREP database are indicated by different colors. Numbers adjacent to sequences correspond to regions shown in panel $A$ and $C$, respectively. (B) Scale bars, 10 kbp; $(D)$ scale bars, $30 \mathrm{kbp}$.

transposons (class II) (Supplemental Information S1; Supplemental Table S7.1). Among the class I elements, Gypsy and Copia LTR retroelements comprised the major component of the repeats, while CACTA DNA elements were highly predominant among class II DNA repeat types. No major differences in the repeat composition of the three genomes were apparent. Compared with Brachypodium distachyon, which has a related but much smaller genome (Vogel et al. 2010), there has been a greater than $100 \times$ increase in repeat content, driven by both class I and class II expansion. The preponderance of CACTA DNA elements in the wheat genome emerged during this massive expansion.

\section{Gene prediction and annotation}

A total of 217,907 loci and 273,739 transcripts were identified from a combination of cross-species protein alignments, 1.5 million high-quality long Pacific Biosciences (PacBio) cDNA reads, and over 3.2 billion RNA-seq read pairs covering a range of tissues and developmental stages (Table 3; Supplemental Information S8).

Loci were identified as coding, long ncRNA, or repeat associated and were classified as high (HC) or low (LC) confidence based on similarity to known plant protein sequences and supporting evidence from wheat transcripts (Supplemental Information S8.5.5). We assigned 104,091 coding genes (154,798 transcripts) as HC, of which 95,827 spanned at least $80 \%$ of the length of the best identified homolog (termed protein rank 1, P1, in the annotation) (Supplemental Fig. S8.1; Supplemental Information S8.5.1). The HC protein-coding set contained 51,851 genes confirmed by a PacBio transcript (Transcript rank 1, T1) and an additional 29,996 genes fully supported by assembled RNA-seq data (T2), providing full transcriptome support for 81,847 (78.63\%) HC genes. Gene predictions were assessed by identifying 2707 single copy genes common to B. distachyon, Oryza sativa, Sorghum bicolor, Setaria italica, and Zea mays. A single orthologous wheat gene was identified for $2686(99.22 \%)$ of these, with $2665(98.45 \%)$

Table 3. Characteristics of predicted high (HC) and low (LC) confidence wheat genes including coding (mRNA) and long noncoding (ncRNA) RNA

\begin{tabular}{|c|c|c|c|c|c|c|}
\hline & All TGAC Models & mRNA HC & mRNA LC & ncRNA HC & ncRNA LC & Repeat-associated \\
\hline Genes & 217,907 & 104,091 & 83,217 & 10,156 & 9933 & 10,510 \\
\hline Transcripts & 273,739 & 154,798 & 85,778 & 11,591 & 10,438 & 11,134 \\
\hline Transcripts per gene & 1.26 & 1.49 & 1.03 & 1.14 & 1.05 & 1.06 \\
\hline Transcript mean cDNA size (bp) & 1766.12 & 2119.52 & 1304.53 & 1368.24 & 1083.98 & 1462.71 \\
\hline Exons per transcript & 4.48 & 5.83 & 2.8 & 2.58 & 2.76 & 2.27 \\
\hline Exons mean size (bp) & 394.15 & 363.73 & 465.27 & 530.25 & 392.24 & 644.09 \\
\hline Transcript mean CDS size (bp) & $1,165.52$ & $1,361.82$ & 839.97 & - & - & 891.05 \\
\hline \multirow[t]{2}{*}{ Mono-exonic transcripts } & 60,322 & 19,034 & 30,479 & 3061 & 3044 & 4704 \\
\hline & $22.04 \%$ & $12.30 \%$ & $35.53 \%$ & $26.41 \%$ & $29.16 \%$ & $42.25 \%$ \\
\hline \multirow{2}{*}{ Genes with alternative splicing } & 32,616 & 28,608 & 2033 & 1037 & 460 & 478 \\
\hline & $14.97 \%$ & $27.48 \%$ & $2.44 \%$ & $10.21 \%$ & $4.63 \%$ & $4.55 \%$ \\
\hline
\end{tabular}

\section{Genome Research}

www.genome.org 
classified as HC and $21(0.78 \%)$ in the LC set. A high coherence in gene length $(r=0.969)$ was found between wheat and B. distachyon proteins (Supplemental Fig. S8.2). These findings show that the HC gene set is robust and establishes a lower bound estimate for the total number of protein-coding genes in wheat. An additional 103,660 loci were defined as LC (i.e., gene models with all their transcripts either having $<60 \%$ protein coverage or lacking wheat transcript support). These include bona fide genes that were fragmented due to breaks in the current assembly, wheat-specific genes, and genes without transcriptome support (Supplemental Table S8.8).

We also identified 10,156 HC noncoding genes with little similarity in protein databases and low protein-coding potential. The majority of these genes are located in intergenic regions (8854, or $87.18 \%$ ), while most of the remaining 1302 are anti-sense to coding genes (1082, or 10.65\%) (see Supplemental Information, section 8. $5.8) ; 5413$ of wheat noncoding genes (53.30\%) were detected in at least one of the two sequenced wheat diploid progenitor species Triticum urartu and Aegilops tauschii (at least 90\% coverage and 90\% identity) (see Supplemental Information S8.5.8).

To obtain additional support for gene predictions, a proteome map was constructed from 27 wheat tissues (Supplemental Information S9). This identified 2,106,323 significant peptide spectrum matches corresponding to 102,379 distinct peptides. Of these, $96.20 \%$ matched HC genes, while $13.29 \%$ were assigned to LC genes. For 56,391 genes (43,431 HC, 12,960 LC), we were able to identify at least one peptide confirming the predicted coding sequence. Due to the hexaploid nature of wheat, only $22.1 \%$ of the peptides could be assigned to a single gene. Applying progressively stricter filters, by requiring at least two or five peptides, confirmed the protein sequence of 30,607 and 17,316 HC genes, respectively; 10,819 genes met the criteria of having support from multiple peptides with at least one uniquely identifying peptide and were considered as unambiguously corroborated by proteomic data. Among the LC genes, only 368 were identified by two or more peptides that did not match any $\mathrm{HC}$ gene, further supporting confidence assignments. Among these, 343 were classified as LC due to having $<60 \%$ the length of the identified homolog, while the remaining 25 genes were classified as LC due to either repeat association or lack of wheat transcript support.

We compared the TGACv1, CSS (The International Wheat Genome Sequencing Consortium 2014), and Chromosome 3B (Choulet et al. 2014) gene models. Of the 100,344 HC genes in the International Wheat Genome Sequencing Consortium (IWGSC) annotation (PGSB/MIPS version 2.2 and INRA version 1.0 from Ensembl release 29), we were able to transfer 97,072 (97\%) to the TGACv1 assembly with stringent alignment parameters (at least 90\% coverage and 95\% identity). Fewer (72\%) of the IWGSC (The International Wheat Genome Sequencing Consortium 2014) LC, unsupported, repeat associated, and noncoding loci could be aligned (at least 90\% coverage and 95\% identity), likely reflecting differences between the assemblies of repeat rich and difficult to assemble regions. Of the TGACv1 HC genes, $61 \%$ overlapped with an aligned IWGSC HC gene and 78\% to the full IWGSC gene set (Supplemental Information S8.5.7). Less agreement was found between TGACv1 LC and ncRNA genes and the IWGSC annotation, with only $8 \%$ overlapping IWGSC HC loci and $40 \%$ overlapping the full IWGSC gene set (Fig. 3A). Of the 22,904 (22\%) HC TGACv1 genes not overlapping a transferred IWGSC gene, $19,810(86 \%)$ had cross-species protein similarity support with 6665 (29\%) fully supported by a PacBio transcript (Fig. 3B). We identified 13,609 TGACv1 genes that were over- lapped by transcripts originating from two or more IWGSC genes in our annotation, indicating that they were likely fragmented in the CSS assembly. In 8175 of these cases (60\%), we were able to find a PacBio read fully supporting our gene model. These differences reflect improvements in contiguity, a more comprehensive representation of the wheat gene space in our assembly, and improved transcriptome support for annotation.

\section{Alternative splicing}

Alternative splicing is an important mRNA processing step that increases transcriptome plasticity and proteome diversity (Staiger and Brown 2013). The TGACv1 annotation includes high-quality alternative splicing variants identified from PacBio transcriptome reads. To provide a more comprehensive representation of alternative splicing, we subsequently integrated transcript assemblies generated from six strand-specific Illumina libraries (Supplemental Information S8.6; Supplemental Table S8.1). This added a further 121,997 transcripts, increasing the number of genes with splice variants from $15 \%$ in the TGACv1 annotation to $31 \%$ in the supplemented set of transcripts (i.e., incorporating Illumina RNA-seq assemblies), as well as increasing the average number of transcripts per gene from 1.26 to 1.88 . When considering only HC genes, the number of alternatively spliced genes was increased from $27.48 \%$ to $48.80 \%$ (2.36 transcripts per gene), similar to that observed in a wide range of plant species (Zhang et al. 2015).

Intron retention (IR) was the prevalent alternative splicing event in wheat (34\%) followed by alternative $3^{\prime}$ splice sites (A3SS; 27\%), exon skipping (ES; 20\%), alternative 5' splice sites (A5SS; 19\%), and mutually exclusive exons (MXE; 0.04\%). This was similar to previous analyses of Chromosome 3B (Pingault et al. 2015), and IR is also predominant in barley (Panahi et al. 2015). Alternative splicing coupled to nonsense mediated decay (NMD) regulates gene expression (Lykke-Andersen and Jensen 2015 ). We found $22 \%$ of all transcripts (17\% of all genes) and $29 \%$ of multiexonic HC protein-coding transcripts (33\% genes) may be potential targets for NMD. IR was the most common splicing event leading to NMD sensitivity, with $40 \%$ of IR transcripts identified as potential NMD targets (34\% ES, 38\% A5SS, 34\% A3SS, 26\% MXE). This suggests a potentially substantial role for alternative splicing/NMD in regulating gene expression in wheat.

\section{Gene families}

HC and LC gene families were analyzed separately using OrthoMCL version 2.0 (Li et al. 2003; Supplemental Figs. S10.1, S10.2). Splice variants were removed from the HC gene data set, keeping the representative transcript for each gene model (see Supplemental Information S8.5.6, S10.1), and data sets were filtered for premature termination codons and incompatible reading frames. For the $\mathrm{HC}$ gene set, a total of 87,519 coding sequences were clustered into 25,132 gene families. The vast majority of $\mathrm{HC}$ gene families contained members from the A, B, and D genomes, consistent with the relatively recent common ancestry of the A and $\mathrm{B}$ genomes and the proposed hybrid origin of the $\mathrm{D}$ genome from ancestral A and B genomes (Marcussen et al. 2014). Subsets of gene families and singleton genes (those not clustered into any family) were classified to identify (1) genes and families that are A, B, or D genome specific; (2) gene families with expanded numbers in one genome; and (3) wheat gene families that are expanded relative to other species. These gene sets were analyzed for overrepresented Gene Ontology (GO) terms, shown in Supplemental File S2. Gene families that were significantly expanded in wheat 
A $\square$ Identical $\square$ Contained $\square$ Structurally different $\square$ Missing

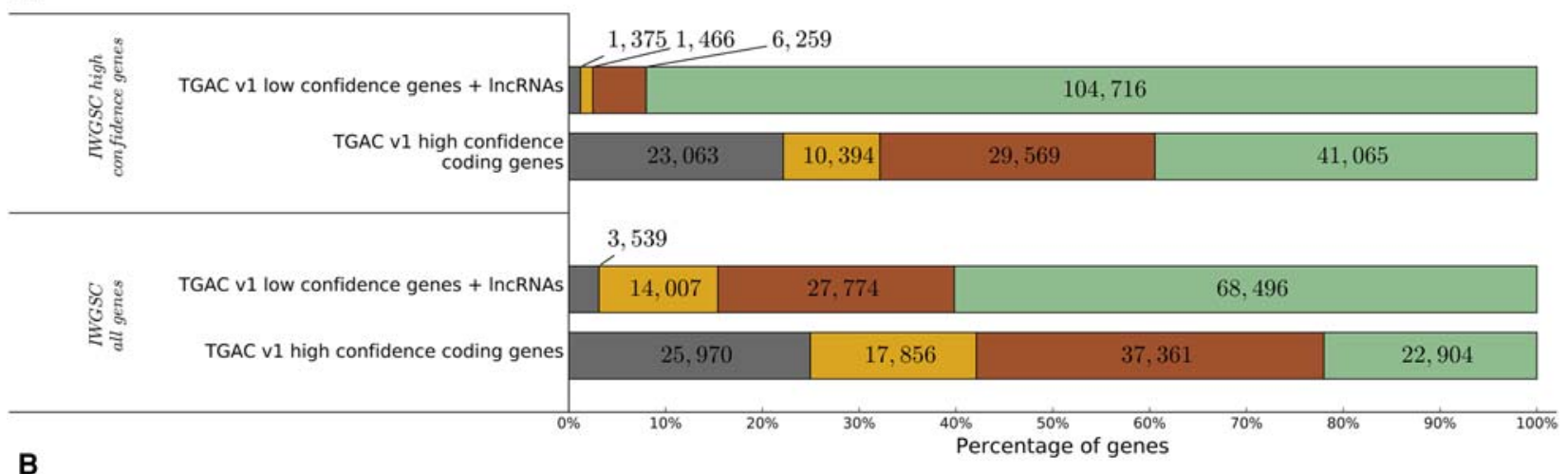

B

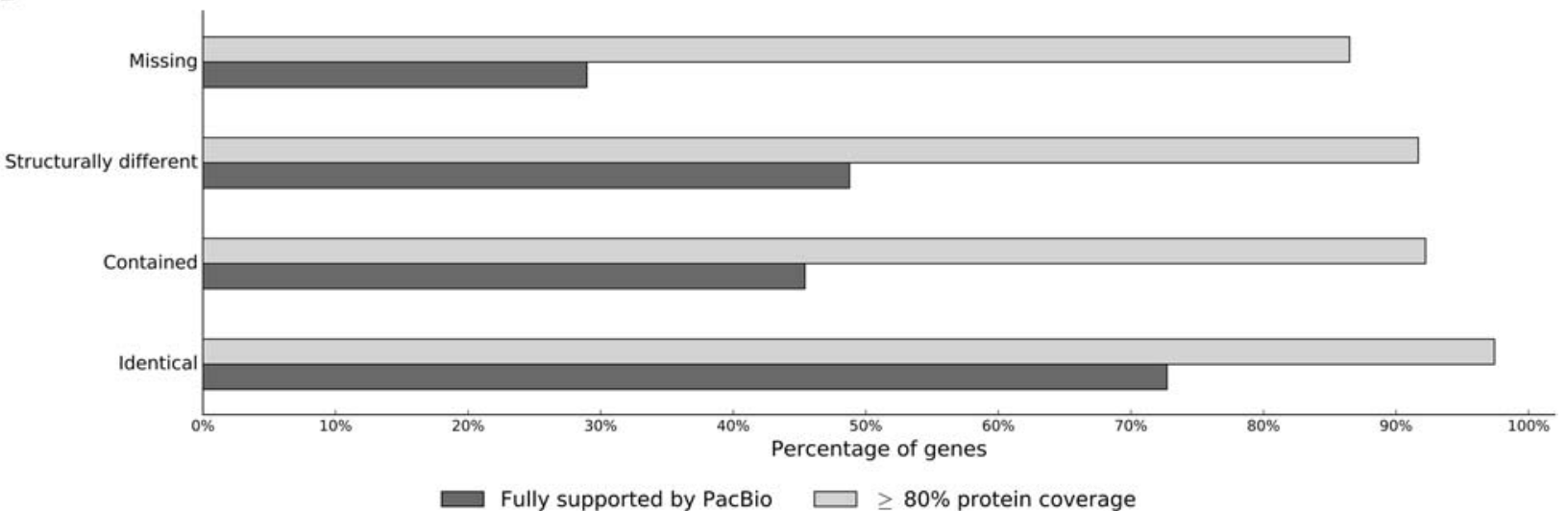

Figure 3. Comparison between IWGSC annotation and TGACV1 high (HC) and low confidence (LC) genes. IWGSC genes were aligned to the TGACV1 assembly (gmap, $\geq 90 \%$ coverage, $\geq 95 \%$ identity) and classified based on overlap with TGACv1 genes. ( $A$ ) Identical indicates shared exon-intron structure; contained, exactly contained within the TGACV1 gene; structurally different, alternative exon-intron structure; and missing, no overlap with IWGSC. (B) Bar plot showing proportion of HC TGACv1 protein-coding genes supported by protein similarity or PacBio data. Genes are classified based on overlap with the full set of IWGSC genes.

compared with Arabidopsis, rice, sorghum, and Brachypodium include those encoding proteins involved in chromosome maintenance and reproductive processes, as well as protein and macromolecule modification and protein metabolism processes. The D genome has expanded gene families encoding phosphorylation, phosphate metabolism, and macromolecule modification activities, while the $\mathrm{B}$ genome has expanded gene families encoding components of chromosome organization, DNA integration and conformation/unwinding, and telomere maintenance. The $\mathrm{B}$ genome is derived from the Sitopsis section of the Triticeae, which has contributed genomes to many polyploid Triticeae species (Riley et al. 1961), suggesting B genomes may have contributed gene functions for establishing and maintaining polyploidy in the Triticeae. This is supported by the location of the major chromosome pairing Ph1 locus on Chromosome 5B (Griffiths et al. 2006).

\section{Genome organization}

A corrected version of the POPSEQ genetic map (Chapman et al. 2015) was used to order TGACv1 scaffolds along chromosomes (Supplemental information S5). This uniquely assigned 128,906 $(17.5 \%)$ of the 735,943 TGACv1 scaffolds to 1051 of 1187 genetic bins (class 1) (Supplemental Information S5) to form the final TGACv1 map. The total length of these scaffolds is $8,551,191,083 \mathrm{bp}$, representing $63.68 \%$ of the TGACv1 assembly and $50.52 \%$ of the 17 -Gbp wheat genome. A further 13,019 (1.77\%) scaffolds were ambiguously assigned to different $\mathrm{cM}$ positions on the same chromosome (class 2), 489 (0.07\%) scaffolds were assigned to homoeologous chromosomes (class 3), and $3320(0.45 \%)$ scaffolds had matching markers with conflicting bin assignment (class 4 ).

The TGACv1 map also assigns unique chromosomal positions to 3927 (3.05\%) scaffolds that were not previously assigned to a chromosome arm (class 5). The CSS-based chromosome arm assignments of $380(0.295 \%)$ class1 scaffolds and $11(0.08 \%)$ class 2 scaffolds disagree with the map-based chromosome assignments (classes 6, 7). A list of scaffold classifications can be found in Supplemental Information S6.

The TGACv1 map encompasses 38,958 of the 53,792 scaffolds containing at least one annotated HC protein-coding gene $(72.42 \%)$, comprising gene sequences of 307,085,968 bp (73.28\% of total predicted gene sequence space). In total, we were able to assign genetic bins to 75,623 (72.65\%) of the HC genes.

Chromosomal locations of related genes were identified by anchoring to the TGACv1 map and are displayed in Figure 4. Analysis of OrthoMCL outlier triads (Supplemental Information S1, sections S6, S10) provided genomic support for known ancestral reciprocal translocations between chromosome arms $4 \mathrm{AL}$ and $5 \mathrm{AL}$, a combination of pericentromeric inversions between chromosome arms $4 \mathrm{AL}$ and $5 \mathrm{AL}$, and a reciprocal exchange 


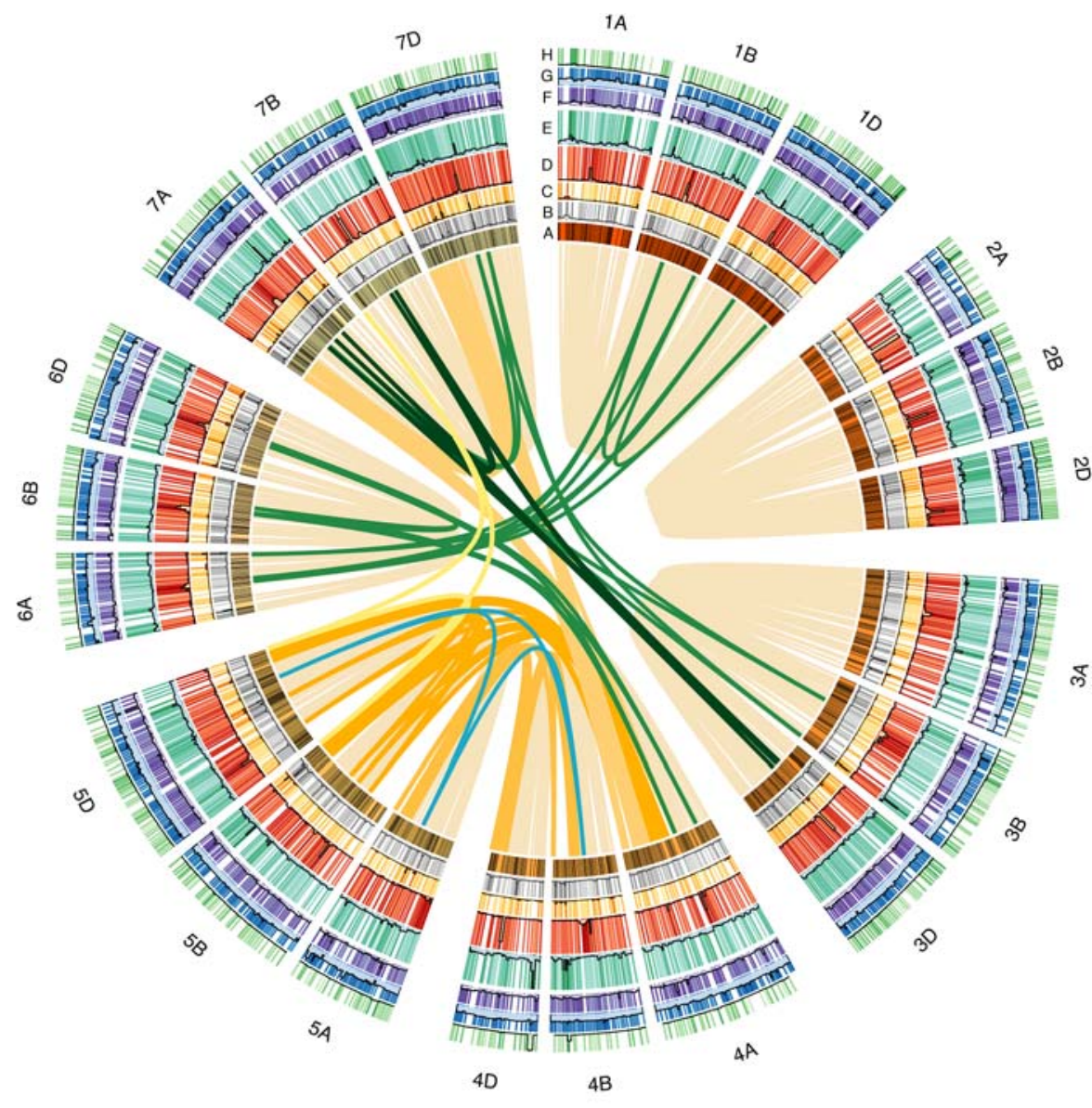

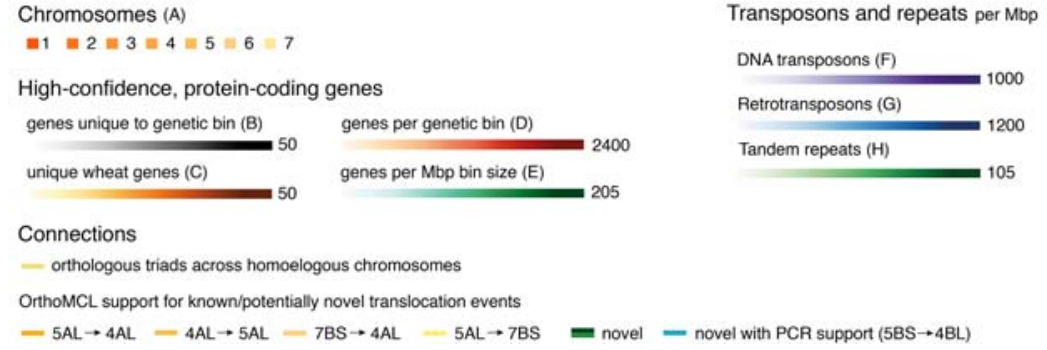

Figure 4. Circular representation of the TGACv1 CS42 assembly. Chromosomes, genetic bins, and genomic features are visualized on the outer rings $(A-H)$ and interchromosomal links identify known and potentially novel translocation events. $(A)$ The seven chromosome groups of the $A, B$, and $D$ genomes, scaled by number of genetic bins (black bands). $(B-H)$ Combined heatmap/histogram representations of genomic features per genetic bin. With the exception of $D$, all counts are normalized by the size of the genetic bin in Mbp, calculated as the total size of all scaffolds assigned to the bin. (B) Distribution of unique genes, i.e., genes that did not have orthologs in a genome-wide OrthoMCL screen. (C) Distribution of wheat-specific genes. $(D, E)$ Number of $\mathrm{HC}$ protein-coding genes. $(F)$ Distribution of DTC, DTM, and DTH DNA transposons (Supplemental Information S1; Supplemental Table S7.1). (G) Distribution of RLX, RLC, RLG, RXX, and RIX retrotransposons. $(H)$ Distribution of tandem duplications. Light yellow links connect homoeologous OrthoMCL triads. Dark yellow-colored links connect genetic bins harboring OrthoMCL outlier triads (Supplemental Information S1, section S6) that identify known translocation events. Dark green links connect genetic bins harboring at least three OrthoMCL outlier triads that may support novel translocation events. The cyan link shows a novel PCR-validated translocation event between Chromosomes 5BS-4BL.

between chromosome arms 4AL and 7BS (Devos et al. 1995). Several putative novel chromosomal translocations were also identified (Fig. 4; Supplemental File S3). As these may have originated in the parental lines used in the POPSEQ map rather than in CS42, nine genes in the predicted translocations (six previously known and three novel) were tested using PCR assays on Chinese Spring chromosomal deletion stocks (Sears et al. 1966). Three known translocation events-4AL-5AL and 7BS-4AL (Devos et al. 1995) and 5AL7BS (Ma et al. 2013) —and one previously unidentified translocation, 5BS-4BL, were validated by PCR assays.

\section{Gene expression}

To explore global gene expression patterns, we mapped multiple wheat RNAseq data sets to the TGACv1 transcriptome (Supplemental Information S1; Supplemental Table S11.1). Seventy-five percent of RNA-seq reads mapped to the TGACv1 transcriptome (Supplemental Information S1; Supplemental Table S11.1), and 78\% of the HC protein-coding transcripts were expressed above the background level of 2 tpm (Wagner et al. 2013). Interestingly, $23 \%$ of the LC genes were also expressed above 2 tpm. Expression levels of genes across chromosomes were similar, with the exception of 19 genetic bins that had increased expression (defined as "hotspots" with a median expression level $>20$ tpm, containing on average 5 genes) across the six tissues examined (Supplemental Information; Supplemental Fig. S11.1). Hotspots tended to be enriched for genes encoding components of the cytoskeleton, ribosome biogenesis, and nucleosome assembly that were expressed at high levels in all tissues. Other notable hotspots were enriched in genes of photosystem I formation in leaf tissues, and nutrient reservoir activity in seed tissues.

The more complete and accurate annotation provided an opportunity to analyze patterns of transcript levels in homoeologous triads. Transcript levels of 9642 triads were analyzed in response to biotic and abiotic stress using publicly available RNA-seq data sets, selected as they all used 7-d-old seedlings, were replicated, and assessed dynamic transcriptional responses to standardized treatments (Supplemental Information S1; Supplemental Table S11.2). Across treatments, $26 \%$ (2424 of 9159) of expressed triads showed higher expression in one or two genomes in at least one stress condition (rather than balanced expression of three genomes) (see Supplemental Information S11.5). Abiotic stress led to more differentially regulated transcripts, compared with biotic stress responses, across all three genomes. To assess the 
conservation of this stress response between homoeologs, we classified each homoeolog as either up-regulated (greater than twofold change, UP), down-regulated (less than 0.5-fold change, DOWN), or flat (between 0.5 -fold to twofold change). We then assessed whether the individual homoeolog response to stress compared with control conditions was consistent (Supplemental Information S1; Supplemental Table S11.3). Eighty percent $( \pm 5.1 \%$ SE) of triads were not differentially expressed in response to the stress treatments and were excluded from further analysis. The most frequent pattern of differential triad expression was a single homoeo$\log$ UP or DOWN, with the other two remaining flat (79\%-99\% across conditions) (Fig. 5). Triads in which either all homoeologs were expressed in the same pattern (" 3 UP" or " 3 DOWN") were rare, as were triads in which homoeologs were expressed in opposite directions. This is consistent with Liu et al. (2015), who identified between $13 \%$ and $41 \%$ of homoeolog triads in which homoeologs did not respond to the same degree in response to stress conditions.

The genomic context of differences in homoeolog expression was explored in genomic regions containing at least five $\mathrm{HC}$ genes in syntenic order on all three genomes, of which at least one homoeolog was expressed over background levels in root, shoot, and endosperm tissue at 10 and $20 \mathrm{~d}$ post anthesis (DPA; DRP000768 and ERP004505) (Supplemental Information S1; Supplemental Table S11.1; Pfeifer et al. 2014). Of the four blocks meeting these criteria, one showed equal expression of all 15 homoeologs in at least one of the tissues, while the other three blocks showed unbalanced expression of at least one homoeolog (Supplemental Information S1; Supplemental Fig. S11.2). All blocks exhibited major structural and promoter sequence differences, as well as variant transcription start sites (Supplemental Information S1; Supplemental Fig. S11.3). These multiple types of genomic differences all have the potential to contribute to unbalanced expression. To facilitate further expression studies the expression atlas at http://www.wheat-expression. com has been updated with the TGACv1 annotation and expression data from 424 RNA samples (Borrill et al. 2015).

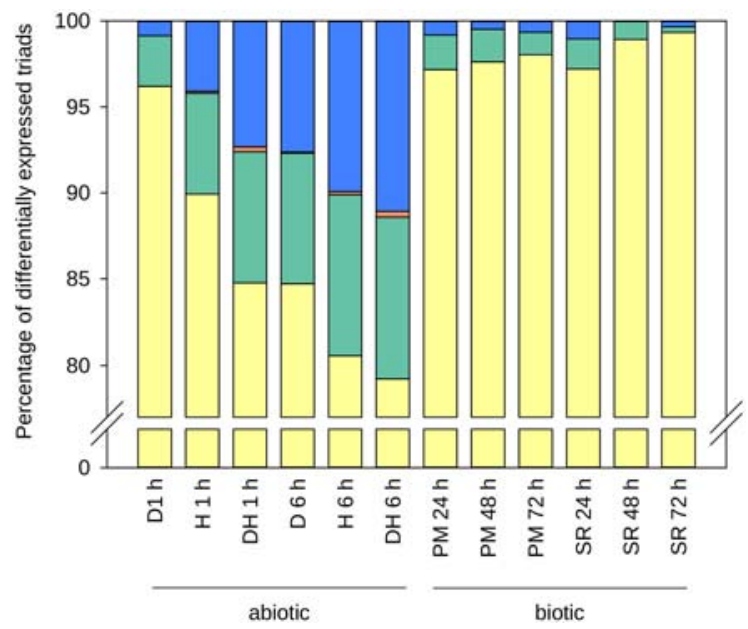

Figure 5. Response of differentially expressed (DE) triads to stress treatments according to the number and pattern of DE homoeologs. Triads were classified as having one homoeolog DE (yellow), two homoeologs DE with same direction of change (green), three homoeologs DE with same direction of change (orange), or opposite direction of change between DE homoeologs (blue). The stresses applied were drought (D), heat $(\mathrm{H})$, drought and heat combined $(\mathrm{DH})$, powdery mildew (PM), and stripe rust (SR), with the duration of stress application indicated in hours (h).

\section{Gene families of agronomic interest}

\section{Wheat disease-resistance genes}

Plant disease-resistance $(R$-) genes termed nucleotide binding siteleucine rich receptors (NBS-LRRs) (Dodds and Rathjen 2010) are challenging to assemble as they are often organized in multigenic clusters with many tandem duplications and rapid pseudogenization. The TGACv1 assembly contains 2595 NBS-containing genes (Table 4) of which 1185 are NBS-LRR genes. Among these, 98\% have complete transcripts compared with only $2 \%$ in the CSS assembly. We also used NLR-parser (Steuernagel et al. 2015) to predict the coiled-coil (CC-) NBS-LRR subclass of $R$-genes. We identified 859 complete CC-NBS-LRR genes supported by specific MEME motifs (Jupe et al. 2012) compared with 225 in the CSS assembly (Table 4). The total of 1185 wheat NBS-NLRs was consistent with that found in diploid wheat progenitors (402 NLRs in T. urartu) and diploid relatives (438 in O. sativa) (Sarris et al. 2016). Nearly $90 \%$ of CS42 R-genes were unambiguously assigned to chromosome arms, and $57 \%(674 / 1185)$ were anchored to the TGACv1 map. The number of $R$-genes per scaffold ranged from one to 31 , compared with only two to three $R$-gene per scaffold in the CSS wheat assembly (The International Wheat Genome Sequencing Consortium 2014). This finding is corroborated by BAC sequence assemblies (Supplemental Information S1; Supplemental Fig. S12.1).

\section{Gluten genes}

Glutens form the major group of grain storage proteins, accounting for $10 \%-15 \%$ of grain dry weight, and confer viscoelastic properties essential for bread-making (Shewry et al. 1995). Gluten genes encode proteins rich in glutamines and prolines that form lowcomplexity sequences composed of PxQ motifs, and occur in tandem repeats in highly complex loci that have posed significant challenges for their assembly and annotation. We characterized the gluten genes in the TGACv1 assembly and showed that most of the known genes were fully assembled. Gluten loci, while still fragmented, exhibit much greater contiguity than in the CSS assembly (The International Wheat Genome Sequencing Consortium 2014) with up to six genes per scaffold (Supplemental Information S1; Supplemental Fig. S12.2). We identified all assembly regions with nucleotide similarity to publicly available gluten sequences, adding an additional 33 gluten genes to the annotation and manually correcting 21 gene models. In total, we identified 105 full-length or partial gluten genes and 13 pseudogenes in the TGACv1 assembly (Table 4; Supplemental information S1, section S12.2).

\section{The gibberellin biosynthetic and signaling pathway}

Mutations in the gibberellin (GA) biosynthetic and signal transduction pathways have been exploited in wheat, where gain-offunction mutations in the GA signaling protein Rht-1 confer GA insensitivity and a range of dwarfing effects. Most modern wheat cultivars carry semi-dominant Rht-1 alleles (Phillips 2016), but these alleles also confer negative pleiotropic effects, including reduced male fertility and grain size. Hence, there is considerable interest in developing alternative dwarfing alleles based on GAbiosynthetic genes such as GA20ox2. A prerequisite for this is access to a complete set of genes encoding the biosynthetic pathway. Figure 6 shows that the TGACv1 assembly contains full-length sequences for 67 of the expected 72 GA pathway genes, in contrast to only 23 genes in the CSS assembly (The International Wheat

\section{Genome Research}

www.genome.org 
Table 4. Disease-resistance and gluten gene repertoires in the TGACv1 assembly

\begin{tabular}{|c|c|c|c|c|c|}
\hline \multicolumn{3}{|l|}{$R$-genes } & \multicolumn{3}{|c|}{ Gluten genes } \\
\hline & CSS & TGACv1 & & CDS & Pseudogenes \\
\hline NBS-containing (Pfam) & 1224 & 2595 & Gliadins & & \\
\hline Fragmented & 1188 & 65 & Alpha & 29 & 9 \\
\hline Complete transcript & 36 & 2530 & Gamma & 18 & 0 \\
\hline No. of scaffolds & 1195 & 1853 & Unknown & 14 & 1 \\
\hline Maximum genes per scaffold & 3 & 31 & Omega & 10 & 0 \\
\hline NBS-LRR (Pfam) & 627 & 1185 & Glutenins & & \\
\hline Partial genes & 611 & 11 & HMW & 6 & 1 \\
\hline Full-length genes & 16 & 1174 & LMW & 16 & 1 \\
\hline No. of scaffolds & 613 & 979 & Prolamins & & \\
\hline Maximum genes per scaffold & 2 & 13 & Avenin & 4 & 0 \\
\hline \multirow{5}{*}{ CC-NBS-LRR (NLR-parser) } & 225 & 859 & Farinin & 4 & 0 \\
\hline & & & Globulin & 2 & 1 \\
\hline & & & Hordein & 1 & 0 \\
\hline & & & Unknown & 1 & 0 \\
\hline & & & Total & 105 & 13 \\
\hline
\end{tabular}

Resistance genes were identified by their characteristic domain architecture (Sarris et al. 2016). Gluten genes were identified by sequence similarity to either a gliadin, glutenin, or generic prolamin class, representing prolamin-like glutens discovered in oat (avenin), wheat (farinin), or barley (hordein). See Supplemental Information, section 12.

Genome Sequencing Consortium 2014). Two paralogs of GA20ox3 on Chromosome 3D are separated by $460 \mathrm{~kb}$, and GA1ox-B1 and GA3ox-B3 are separated by $3.2 \mathrm{~kb}$, suggesting common ancestry of these two enzymes with different catalytic activities (Pearce et al. 2015).

\section{Discussion}

Access to a complete and robust wheat genome assembly is essential for the continued improvement of wheat, a staple crop of global significance with $728 \mathrm{M}$ tonnes produced in 2014 (http://fenix. fao.org/faostat/beta/en/\#home). The capacity to assemble and annotate wheat genomes accurately, rapidly, and cost-effectively addresses key social, economic, and academic priorities by facilitating trait analyses, by exploiting diverse germplasm resources, and by accelerating plant breeding. However, polyploidy and the extensive repeat regions in wheat have limited the completeness of previous assembly efforts (Brenchley et al. 2012; The International Wheat Genome Sequencing Consortium 2014; Chapman et al. 2015), reducing their utility.

Here we report a much more complete wheat genome assembly, representing $\sim 80 \%$ of the $17-\mathrm{Gb}$ genome in large scaffolds. We combined high-quality PCR-free libraries and precisely sizeselected LMP libraries (Heavens et al. 2015) with the w2rap assembly software (Clavijo et al. 2017) to generate contiguous and complete assemblies from relatively low (about 33x) Illumina PE read coverage and LMP libraries. The contiguity of the TGACv1 assembly allowed us to create a greatly improved gene annotation supported by extensive transcriptome data. Over $78 \%$ of the 104,091 HC protein-coding genes are fully supported by RNA-seq data. These improvements identified 22,904 genes that were absent from previous wheat gene sets Bioactive GAs are boxed in red.
(The International Wheat Genome Sequencing Consortium 2014; Choulet et al. 2014), almost all of which have a homolog in other species (Fig. 3B). The robustness of the annotation is further supported by the use of high-quality PacBio data and agreement with proteomic data, with $42 \%$ of the HC gene models supported by sequenced peptides. This new wheat gene set provides an improved foundation for wheat research. Finally, incorporation of strand-specific Illumina RNA-seq libraries into the annotation showed that nearly half of the HC genes were alternatively spliced, in line with observations in many other plants (Zhang et al. 2015).

A well-defined gene set in large sequence scaffolds is an essential foundation for trait analyses in wheat. We identified the complement of disease-resistance genes, gluten protein genes that confer nutritional and bread-making quality of wheat grains, and the set of GA biosynthetic and signal transduction genes that are important determinants of crop height and yield. An accurate gene set is also essential for understanding expression of gene families in complex allopolyploid genomes. We observed that $20 \%$ of homoeologous triads showed differential expression in seedling

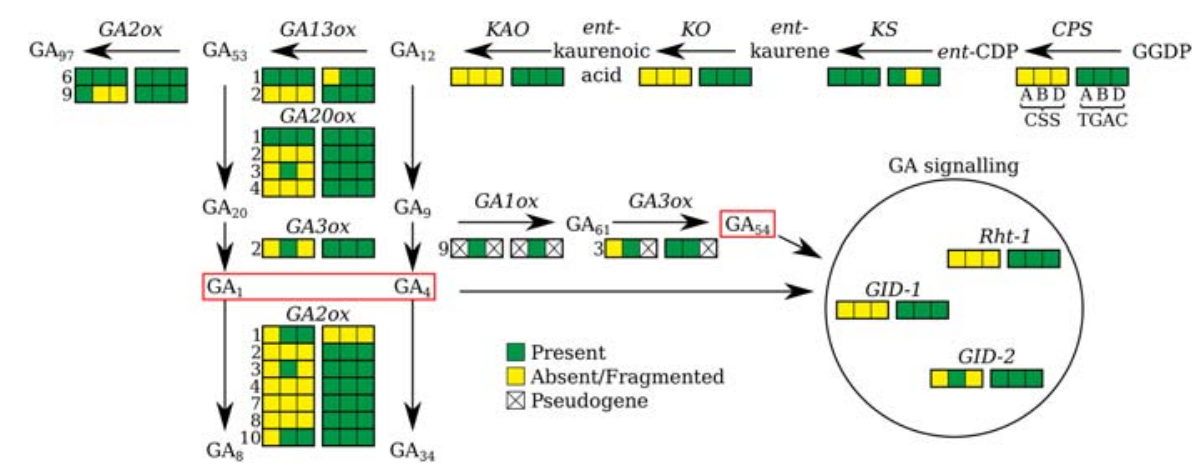

Figure 6. Genes encoding the gibberellin (GA) biosynthetic and signaling pathway in bread wheat. The GA biosynthesis, inactivation, and signal transduction pathway, illustrating the representation of the gene sequences in CSS and TGACV1 assemblies. If more than one paralog is known for a gene, its number according to the classification by Pearce et al. (2015) is indicated on the left of the box. 
leaves subject to biotic and abiotic stress conditions. This is consistent with coexpression analyses in developing grains (Pfeifer et al. 2014), where most differentially expressed genes were single homoeologs that were up/down-regulated. Taken together, these results identify widespread subfunctionalization of homoeologous genes due to differential regulation. The new assembly and annotation will enable the identification of multiple sequence differences in promoters, transcription start sites, gene splicing, and other features among strict homoeologs, providing a foundation for systematic analyses of the causes of these differences.

Generating complete and accurate wheat genome assemblies is essential for capturing the full range of genetic variation in wheat genomes. By identifying this variation, genomics will directly facilitate trait analyses and accelerate plant breeding. Our rapid, accurate, and cost-effective assembly approach is suitable for assembling multiple wheat and other Triticeae genomes in robust and comparable ways, using relatively inexpensive sequencing technologies based on PCR-free libraries and opensource software. We anticipate that researchers with access to suitable computational infrastructure will use the approaches described here to sequence multiple wheat varieties, including elite varieties, unimproved landraces, and progenitor species. These assemblies will reveal a wide spectrum of genetic variation, including large-scale structural changes such as translocations and chromosome additions that are known to play a major role in the adaptation of the wheat crop to different growing environments. By adopting this pan-genomics approach, we will enrich our understanding of complex genome evolution and the plasticity of genome regulation and empower new approaches to wheat improvement.

\section{Methods}

\section{DNA library preparation and sequencing}

A full description of the DNA preparation and sequencing methods is in Supplemental Information. PCR-free PE libraries were sequenced using $2 \times 250$-bp reads on HiSeq2500 platforms for contig generation. TALL libraries and Nextera LMP libraries (Heavens et al. 2015) were used for scaffolding. Insert size distributions (Supplemental Information S1; Supplemental Figs. S4.1-S4.3) were checked by mapping to the CS42 Chromosome 3B pseudomolecule (Choulet et al. 2014) using the DRAGEN coprocessor (http://www.edicogenome.com/dragen/).

\section{Genome assembly}

Assembly was performed using the Wheat/Whole-Genome Robust Assembly Pipeline, w2rap (Clavijo et al. 2017). It combines the w2rap-contigger, based on DISCOVAR de novo (Weisenfeld et al. 2014), an LMP preparation approach based on FLASH (Magoc and Salzberg 2011) and Nextclip (Leggett et al. 2014), and scaffolding with SOAPdenovo2 (Luo et al. 2012). The w2rap-contigger takes advantage of DISCOVAR (Weisenfeld et al. 2014; Love et al. 2016) algorithms to preserve sequence variation during assembly but has been further developed to enable processing of much larger data volumes and complex genomic repeats. The pairedend read data set was assembled into contigs on a SGI UV200 machine with 7TB of shared RAM. The contig assembly took $38 \mathrm{~d}$ using 64 cpus, with the default settings of the w2rap-contigger from https://github.com/bioinfologics/w2rap-contigger/releases/ tag/CS42_TGACv1. Newer versions of w2rap can achieve similar results in half the time or less, using close to half the memory. Scaffolding with the LMP data took a total of $10 \mathrm{~d}$ and was execut- ed on the same hardware but used 128 cpus and $<1$ TB of RAM. Contigs were scaffolded using the PE, LMP, and TALL reads and the SOAPdenovo2 (Luo et al. 2012) prepare $\rightarrow$ map $\rightarrow$ scaffold pipeline, run at $k=71$. Contigs and scaffolds were quality controlled using KAT spectra-cn plots (Mapleson et al. 2017) to assess motif representation.

\section{Gene annotation}

A high-quality gene set for wheat was generated using a custom pipeline integrating wheat-specific transcriptomic data, protein similarity, and evidence-guided gene predictions generated with AUGUSTUS (Stanke and Morgenstern 2005). Full methods are in Supplemental Information S8. RNA-seq reads (ERP004714, ERP004505, and 250-bp PE strand-specific reads from six different tissues) were assembled using four alternative assembly methods (Trapnell et al. 2010; Haas et al. 2013; Pertea et al. 2015; Song et al. 2016) and integrated with PacBio transcripts into a coherent and nonredundant set of models using Mikado (https://github. com/lucventurini/mikado). PacBio reads were then classified according to protein similarity and a subset of high-quality (e.g., full length, canonical splicing, nonredundant) transcripts used to train an AUGUSTUS wheat-specific gene prediction model. AUGUSTUS was then used to generate a first draft of the genome annotation, using as input Mikado-filtered transcript models, reliable junctions identified with Portcullis (https://github.com/ maplesond/portcullis), and peptide alignments of proteins from five close wheat relatives (B. distachyon, maize, rice, $S$. bicolor, and $S$. italica). This draft annotation was refined by correcting probable gene fusions, missing loci and alternative splice variants. The annotation was functionally annotated, and all loci were assigned a confidence rank based on their similarity to known proteins and their agreement with transcriptome data.

\section{Data access}

All data generated in this study have been submitted to the European Nucleotide Archive (ENA; http://www.ebi.ac.uk/ena) under accession numbers PRJEB15378, PRJEB15378 (PE and LMP reads used for genome assembly and scaffolding), PRJEB11773 (genome assembly), and PRJEB15048 (Illumina and PacBio reads used for genome annotation). The assembly and annotation are available in Ensembl Plants (release 32; Ensembl Plants, http://plants. ensembl.org/Triticum_aestivum/Info/Index) and from the Earlham Institute Open Data site (EI; http://opendata.earlham.ac .uk/Triticum_aestivum/TGAC/v1/). BLAST services for these data sets are available via Grassroots Genomics (Grassroots; https:// wheatis.tgac.ac.uk/grassroots-portal/blast).

\section{Acknowledgments}

We thank Jane Rogers and Mario Caccamo for initial discussions and planning and Burkhard Steuernagel for assistance with NLRparser. This work was funded by a Biotechnology and Biological Sciences Research Council (BBSRC) strategic LOLA Award to M. W.B. and C.U. (BB/J003557/1), M.D.C. (BB/J003743/1), P.J.K. (BB/J00328X/1), and A.L.P. (BB/J003913/1); a BBSRC Anniversary Future Leader Fellowship (BB/M014045/1) to P.B.; BBSRC Institute Strategic Programme Grants GRO (BB/J004588/1) to M.W.B. and C.U.; "2020 Wheat" (BBS/E/C/00005202) to A.L.P.; and Bioinformatics BB/J004669/1 to F.D.P. The German Ministry of Education and Research (BMBF) grant 031A536 "de.NBI" supported M.S., and the Australian Research Council (LP120200102, CE140100008) and Agilent Technologies Australia supported A. H.M. Next-generation sequencing and library construction was

\section{Genome Research}

www.genome.org 
delivered via the BBSRC National Capability in Genomics (BB/ J010375/1) at the Earlham Institute (EI; formerly The Genome Analysis Centre, Norwich), by members of the Platforms and Pipelines Group. Open data access and BLAST databases and service are provided by the EI Data Infrastructure group.

\section{References}

The 1000 Genomes Project Consortium. 2010. A map of human genome variation from population-scale sequencing. Nature 467: 1061-1073.

Abdel-Ghany SE, Hamilton M, Jacobi JL, Ngam P, Devitt N, Schilkey F, BenHur A, Reddy ASN. 2016. A survey of the sorghum transcriptome using single-molecule long reads. Nat Commun 7: 11706.

Aird D, Ross MG, Chen W-S, Danielsson M, Fennell T, Russ C, Jaffe DB Nusbaum C, Gnirke A. 2011. Analyzing and minimizing PCR amplification bias in Illumina sequencing libraries. Genome Biol 12: R18.

Berthelot C, Brunet F, Chalopin D, Juanchich A, Bernard M, Noël B, Bento P, Da Silva C, Labadie K, Alberti A, et al. 2014. The rainbow trout genome provides novel insights into evolution after whole-genome duplication in vertebrates. Nat Commun 5: 3657.

Bickhart DM, Rosen BD, Koren S, Sayre BL, Hastie AR, Chan S, Lee J, Lam ET, Liachko I, Sullivan ST, et al. 2017. Single-molecule sequencing and chromatin conformation capture enable de novo reference assembly of the domestic goat genome. Nat Genet (in press). doi: 10.1038/ng.3802.

Bishara A, Liu Y, Weng Z, Kashef-Haghighi D, Newburger DE, West R, Sidow A, Batzoglou S. 2015. Read clouds uncover variation in complex regions of the human genome. Genome Res 25: 1570-1580.

Blanc G, Wolfe KH. 2004. Widespread paleopolyploidy in model plant species inferred from age distributions of duplicate genes. Society 16: 1667-1678.

Borrill P, Adamski N, Uauy C. 2015. Genomics as the key to unlocking the polyploid potential of wheat. New Phytologist 208: 1008-1022.

Brenchley R, Spannagl M, Pfeifer M, Barker GLA, D'Amore R, Allen AM, McKenzie N, Kramer M, Kerhornou A, Bolser D, et al. 2012. Analysis of the bread wheat genome using whole-genome shotgun sequencing. Nature 491: 705-710.

Chaisson MJP, Wilson RK, Eichler EE. 2015. Genetic variation and the de novo assembly of human genomes. Nat Rev Genet 16: 627-640.

Chapman JA, Ho I, Sunkara S, Luo S, Schroth GP, Rokhsar DS. 2011. Meraculous: de novo genome assembly with short paired-end reads. PLoS One 6: e23501.

Chapman JA, Mascher M, Buluc A, Barry K, Georganas E, Session A, Strnadova V, Jenkins J, Sehgal S, Oliker L, et al. 2015. A whole-genome shotgun approach for assembling and anchoring the hexaploid bread wheat genome. Genome Biol 16: 26.

Choulet F, Alberti A, Theil S, Glover N, Barbe V, Daron J, Pingault L, Sourdille P, Couloux A, Paux E, et al. 2014. Structural and functional partitioning of bread wheat chromosome 3B. Science 345: 1249721.

Clavijo B, Garcia Accinelli G, Wright J, Heavens D, Barr K, Yanes L, Di Palma F. 2017. W2RAP: a pipeline for high quality, robust assemblies of large complex genomes from short read data. bioRxiv 2017: 110999.

Devos KM, Dubcovsky J, Dvořák J, Chinoy CN, Gale MD. 1995. Structural evolution of wheat chromosomes $4 \mathrm{~A}, 5 \mathrm{~A}$, and $7 \mathrm{~B}$ and its impact on recombination. Theor Appl Genet 91: 282-288.

Dodds PN, Rathjen JP. 2010. Plant immunity: towards an integrated view of plant-pathogen interactions. Nat Rev Genet 11: 539-548.

Gan X, Stegle O, Behr J, Steffen JG, Drewe P, Hildebrand KL, Lyngsoe R, Schultheiss SJ, Osborne EJ, Sreedharan VT, et al. 2011. Multiple reference genomes and transcriptomes for Arabidopsis thaliana. Nature 477: $419-423$.

Gnerre S, MacCallum I, Przybylski D, Ribeiro FJ, Burton JN, Walker BJ, Sharpe T, Hall G, Shea TP, Sykes S, et al. 2011. High-quality draft assemblies of mammalian genomes from massively parallel sequence data. Proc Natl Acad Sci 108: 1513-1518.

Gordon D, Huddleston J, Chaisson MJP, Hill CM, Kronenberg ZN, Munson KM, Malig M, Raja A, Fiddes I, Hillier LW, et al. 2016. Long-read sequence assembly of the gorilla genome. Science 352: aae0344.

Griffiths S, Sharp R, Foote TN, Bertin I, Wanous M, Reader S, Colas I, Moore G. 2006. Molecular characterization of Ph1 as a major chromosome pairing locus in polyploid wheat. Nature 439: 749-752.

Haas BJ, Papanicolaou A, Yassour M, Grabherr M, Blood PD, Bowden J, Couger MB, Eccles D, Li B, Lieber M, et al. 2013. De novo transcript sequence reconstruction from RNA-seq using the trinity platform for reference generation and analysis. Nat Protoc 8: 1494-1512.

Heavens D, Accinelli GG, Clavijo B, Clark MD. 2015. A method to simultaneously construct up to 12 differently sized Illumina Nextera long mate pair libraries with reduced DNA input, time, and cost. Biotechniques 59: $42-45$.
The International Wheat Genome Sequencing Consortium. 2014. A chromosome-based draft sequence of the hexaploid bread wheat (Triticum aestivum) genome. Science 345: 1251788.

Jupe F, Pritchard L, Etherington GJ, MacKenzie K, Cock PJ, Wright F, Sharma SK, Bolser D, Bryan GJ, Jones JD, et al. 2012. Identification and localisation of the NB-LRR gene family within the potato genome. $B M C$ Genomics 13: 75 .

Kozarewa I, Ning Z, Quail MA, Sanders MJ, Berriman M, Turner DJ. 2009. Amplification-free Illumina sequencing-library preparation facilitates improved mapping and assembly of $(\mathrm{G}+\mathrm{C})$-biased genomes. Nat Methods 6: 291-295.

Lam HYK, Clark MJ, Chen R, Chen R, Natsoulis G, O'Huallachain M, Dewey FE, Habegger L, Ashley EA, Gerstein MB, et al. 2011. Performance comparison of whole-genome sequencing platforms. Nat Biotechnol 30: 78-82.

Leggett RM, Clavijo BJ, Clissold L, Clark MD, Caccamo M. 2014. NextClip: an analysis and read preparation tool for Nextera Long Mate Pair libraries. Bioinformatics 30: $566-568$.

Li L. 2003. OrthoMCL: identification of ortholog groups for eukaryotic genomes. Genome Res 13: 2178-2189.

Lieberman-Aiden E, van Berkum NL, Williams L, Imakaev M, Ragoczy T, Telling A, Amit I, Lajoie BR, Sabo PJ, Dorschner MO, et al. 2009. Comprehensive mapping of long-range interactions reveals folding principles of the human genome. Science (New York NY.) 326: 289-293.

Liu Z, Xin M, Qin J, Peng H, Ni Z, Yao Y, Sun Q. 2015. Temporal transcriptome profiling reveals expression partitioning of homeologous genes contributing to heat and drought acclimation in wheat (Triticum aestivum L.). BMC Plant Biol 15: 152.

Love RR, Weisenfeld NI, Jaffe DB, Besansky NJ, Neafsey DE. 2016. Evaluation of DISCOVAR de novo using a mosquito sample for cost-effective short-read genome assembly. BMC Genomics 17: 187.

Luo R, Liu B, Xie Y, Li Z, Huang W, Yuan J, He G, Chen Y, Pan Q, Liu Y, et al. 2012. SOAPdenovo2: an empirically improved memory-efficient shortread de novo assembler. Gigascience 1: 18.

Lykke-Andersen S, Jensen TH. 2015. Nonsense-mediated mRNA decay: an intricate machinery that shapes transcriptomes. Nat Rev Mol Cell Biol 16: $665-677$.

Ma J, Stiller J, Berkman PJ, Wei Y, Rogers J, Feuillet C, Dolezel J, Mayer KF, Eversole K, Zheng Y-L, et al. 2013. Sequence-based analysis of translocations and inversions in bread wheat (Triticum aestivum L.). PLoS One 8: e79329.

Magoc T, Salzberg SL. 2011. FLASH: fast length adjustment of short reads to improve genome assemblies. Bioinformatics 27: 2957-2963.

Mapleson D, Garcia Accinelli G, Kettleborough G, Wright J, Clavijo BJ. 2017. KAT: a K-mer analysis toolkit to quality control NGS datasets and genome assemblies. Bioinformatics 33: 574-576.

Marcussen T, Sandve SR, Heier L, Spannagl M, Pfeifer M, Jakobsen KS, Wulff $\mathrm{BBH}$, Steuernagel B, Mayer KFX, Olsen O-A, et al. 2014. Ancient hybridizations among the ancestral genomes of bread wheat. Science 345: 1250092.

Moore G, Devos K, Wang Z, Gale M. 1995. Cereal genome evolution: grasses, line up and form a circle. Curr Biol 5: 737-739.

Mostovoy Y, Levy-Sakin M, Lam J, Lam ET, Hastie AR, Marks P, Lee J, Chu C, Lin C, Džakula Ž, et al. 2016. A hybrid approach for de novo human genome sequence assembly and phasing. Nat Methods 13: 587-590.

Panahi B, Mohammadi SA, Khaksefidi RE, Fallah Mehrabadi J, Ebrahimie E 2015. Genome-wide analysis of alternative splicing events in Hordeum vulgare: Highlighting retention of intron-based splicing and its possible function through network analysis. FEBS Lett 589: 3564-3575.

Pearce S, Huttly AK, Prosser IM, Li Y-d, Vaughan SP, Gallova B, Patil A, Coghill JA, Dubcovsky J, Hedden P, et al. 2015. Heterologous expression and transcript analysis of gibberellin biosynthetic genes of grasses reveals novel functionality in the GA3ox family. BMC Plant Biol 15: 130.

Pendleton M, Sebra R, Pang AWC, Ummat A, Franzen O, Rausch T, Stütz AM, Stedman W, Anantharaman T, Hastie A, et al. 2015. Assembly and diploid architecture of an individual human genome via singlemolecule technologies. Nat Methods 12: 780-786.

Pertea M, Pertea GM, Antonescu CM, Chang T-C, Mendell JT, Salzberg SL. 2015. StringTie enables improved reconstruction of a transcriptome from RNA-seq reads. Nat Biotechnol 33: 290-295.

Pfeifer M, Kugler KG, Sandve SR, Zhan B, Rudi H, Hvidsten TR, International Wheat Genome Sequencing Consortium, Mayer KFX, Olsen O-A. 2014. Genome interplay in the grain transcriptome of hexaploid bread wheat. Science 345: 1250091.

Phillips AL. 2016. Genetic control of gibberellin metabolism and signalling in crop improvement. In Annual plant reviews, Vol. 49, pp. 405-430. John Wiley \& Sons, Chichester, UK.

Pingault L, Choulet F, Alberti A, Glover N, Wincker P, Feuillet C, Paux E. 2015. Deep transcriptome sequencing provides new insights into the structural and functional organization of the wheat genome. Genome Biol 16: 29. 
Putnam NH, O'Connell BL, Stites JC, Rice BJ, Blanchette M, Calef R, Troll CJ, Fields A, Hartley PD, Sugnet CW, et al. 2016. Chromosome-scale shotgun assembly using an in vitro method for long-range linkage. Genome Res 26: 342-350.

Riley R, Kimber G, Chapman V. 1961. Origin of genetic control of diploidlike behavior of polyploid wheat. J Heredity 52: 22-25.

Sarris PF, Cevik V, Dagdas G, Jones JDG, Krasileva KV. 2016. Comparative analysis of plant immune receptor architectures uncovers host proteins likely targeted by pathogens. BMC Biol 14: 8 .

Sears ER. 1966. Nullisomic-tetrasomic combinations in hexaploid wheat. In Chromosome manipulations and plant genetics, pp. 29-45. Springer, Boston, MA.

Shewry PR, Tatham AS, Barro F, Barcelo P, Lazzeri P. 1995. Biotechnology of breadmaking: unraveling and manipulating the multi-protein gluten complex. Biotechnology 13: 1185-1190.

Song L, Sabunciyan S, Florea L. 2016. CLASS2: accurate and efficient splice variant annotation from RNA-seq reads. Nucleic Acids Res 44: e98.

Staiger D, Brown JWS. 2013. Alternative splicing at the intersection of biological timing, development, and stress responses. Plant Cell 25: 3640-3656.

Stanke M, Morgenstern B. 2005. AUGUSTUS: a web server for gene prediction in eukaryotes that allows user-defined constraints. Nucleic Acids Res 33: W465-W467.

Steuernagel B, Jupe F, Witek K, Jones JDG, Wulff BBH. 2015. NLR-parser: rapid annotation of plant NLR complements. Bioinformatics 31: $1665-1667$

Trapnell C, Williams BA, Pertea G, Mortazavi A, Kwan G, van Baren MJ, Salzberg SL, Wold BJ, Pachter L. 2010. Transcript assembly and quanti- fication by RNA-seq reveals unannotated transcripts and isoform switching during cell differentiation. Nat Biotechnol 28: 511-515.

Treangen TJ, Salzberg SL. 2012. Repetitive DNA and next-generation sequencing: computational challenges and solutions. Nat Rev Genet 13: 36-46.

Vogel JP, Garvin DF, Mockler TC, Schmutz J, Rokhsar D, Bevan MW, Barry K, Lucas S, Harmon-Smith M, Lail K, et al. 2010. Genome sequencing and analysis of the model grass Brachypodium distachyon. Nature 463: 763-768.

Wagner GP, Kin K, Lynch VJ. 2013. A model based criterion for gene expression calls using RNA-seq data. Theory Biosci 132: 159-164.

Weisenfeld NI, Yin S, Sharpe T, Lau B, Hegarty R, Holmes L, Sogoloff B, Tabbaa D, Williams L, Russ C, et al. 2014. Comprehensive variation discovery in single human genomes. Nat Genet 46: 1350-1355.

Weisenfeld NI, Kumar V, Shah P, Church D, Jaffe DB. 2016. Direct determination of diploid genome sequences. Genome Res (this issue). doi: 10.1101/gr.214874.116.

Zhang C, Yang H, Yang H. 2015. Evolutionary character of alternative splicing in plants. Bioinformatics Biol Insights 9: 47-52.

Zimin AV, Puiu D, Luo M-C, Zhu T, Koren S, Marcais G, Yorke JA, Dvorak J, Salzberg SL. 2017. Hybrid assembly of the large and highly repetitive genome of Aegilops tauschii, a progenitor of bread wheat, with the megareads algorithm. Genome Res (this issue). doi: 10.1101/gr.213405.116.

Received October 13, 2016; accepted in revised form March 14, 2017. 


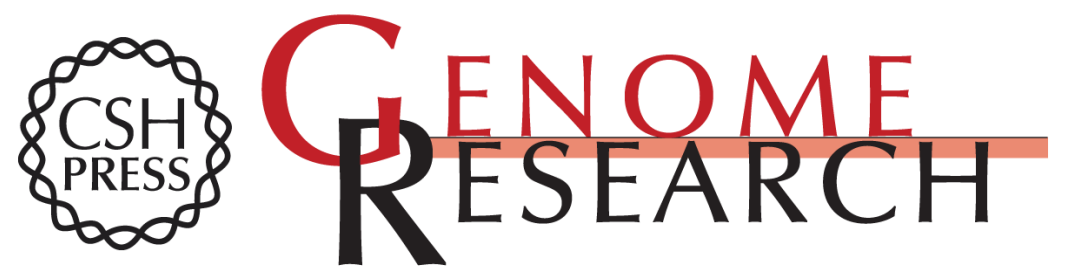

\section{An improved assembly and annotation of the allohexaploid wheat genome identifies complete families of agronomic genes and provides genomic evidence for chromosomal translocations}

Bernardo J. Clavijo, Luca Venturini, Christian Schudoma, et al.

Genome Res. 2017 27: 885-896 originally published online April 18, 2017

Access the most recent version at doi:10.1101/gr.217117.116

Supplemental
Material http://genome.cshlp.org/content/suppl/2017/04/04/gr.217117.116.DC1

References This article cites 62 articles, 12 of which can be accessed free at:

http://genome.cshlp.org/content/27/5/885.full.html\#ref-list-1

Open Access Freely available online through the Genome Research Open Access option.

Creative This article, published in Genome Research, is available under a Creative

Commons Commons License (Attribution 4.0 International), as described at

License http://creativecommons.org/licenses/by/4.0/.

Email Alerting Receive free email alerts when new articles cite this article - sign up in the box at the Service top right corner of the article or click here.

\section{Affordable, Accurate Sequencing.}

To subscribe to Genome Research go to:

https://genome.cshlp.org/subscriptions 